\title{
The PHO pathway involved in phosphate metabolism in Yeast for efficient phosphorus removal
}

\author{
Mengfei $\mathrm{Hu}^{1}$, Liping Qiu ${ }^{1, *}$, and Yan Wang ${ }^{1}$ \\ ${ }^{1}$ School of Civil Engineering and Architecture, University of Jinan, 336 Nanxinzhuang West Road, Jinan, 250022, China.
}

\begin{abstract}
Phosphorus is one of the essential elements needed for the growth and reproduction of any organism. To improve the efficiency of biological phosphorus removal in sewage, it is very important to grasp the precise mechanism of biological phosphorus removal. Yeast is a single cell fungus and has a unique advantage in sewage treatment. Recent studies in the different types of yeast have revealed that there is a phosphate-responsive signal transduction (PHO) pathway to regulate phosphate-responsive genes for controlling phosphate absorption. In this review, the metabolic mechanisms and protein-protein interactions associated with the PHO pathway are highlighted firstly, and then several examples about improving the phosphorus removal efficiency of sewage by inducing gene mutation in yeast phosphorus metabolism was introduced. The aim is to provide new ideas for the realization of high-efficiency phosphorus recovery in nature.
\end{abstract}

\section{Introduction}

Phosphorus is a non-renewable and one-way recycling limited resource. China's phosphate rock resources are listed as one of the major mineral resources that cannot meet the national economic development needs after 2010 [1]. With the development of the country and the continuous growth of industrial enterprises, more and more high-phosphorus wastewater is discharged into natural water bodies, resulting in the proliferation of algae, the lack of dissolved oxygen, and the serious eutrophication of water bodies. Therefore, it is quite meaningful to study how to remove and recycle phosphorus from waste water.

At the same time, phosphorus is an essential element for the formation of nucleic acids, phospholipids, polysaccharides, and proteins in all living organisms. It plays a crucial role in photosynthesis and some regulatory processes of enzymes and is an indispensable macro for the growth and reproduction of organisms [2, 3]. Plants in the absence of inorganic phosphate (Pi), growth and production will decline [4]. Fungi in the low phosphorus conditions will reduce its metabolic activity, and even face death [5]. Therefore, it is possible to use the microbial metabolic activity to achieve the removal of phosphorus in the sewage, and biological phosphorus removal has become the most widely used method of removing phosphorus from wastewater.

\section{Yeast}

Yeast belongs to single-celled fungi, which has the advantages of osmotic pressure resistance, acid resistance, high temperature resistance, high metabolic efficiency and high environmental adaptability [6]. At present, yeast has been widely used in the production of biofuels, chemicals, and wastewater treatment $[7,8]$. In order to adapt to the external environment changes, yeast cells have a good enzyme system to maintain cell survival, and this adaptation often occurs through transcription and translation changes [9]. Yeast has a PHO pathway to regulate the expression of phosphorusrespond genes to adapt the change of external phosphorus concentration, so that the yeast can absorb a large amount of phosphorus in the environment of phosphorus deficiency [10]. In order to cultivate Yeastengineered strains which can be used to remove phosphorus from wastewater, the most important is to accurately regulate the expression of genes involved in environmental responses [11].

\section{The PHO regulation pathway}

\subsection{The PHO pathway}

Phosphate-responsive signal transduction (PHO) pathway, is known to regulate the expression of several phosphate-responsive genes, in order to keep intracellular phosphate concentration stable. In recent years, relevant scholars have continuously studied the PHO pathways of different types of yeast such as Cryptococcus neoformans [12, 13], C. glabrata [14], C. albicans [15], and Budding Yeast [16]. Studies have shown that yeast cells secrete a large amount of acid phosphatase and phosphate transporter under phosphatelimiting conditions. Kinases and transcription factors (TFs) are key regulators of important signalling

\footnotetext{
Corresponding author: lipingqiu@163.com
} 
pathways [17]. Acid phosphatase hydrolyzes extracellular phosphate compounds into inorganic phosphate granules [4], phosphate transporters carry inorganic phosphate granules into the body and store them in the form of polyphosphates as an emergency repository for phosphates [16]; this activity is inhibited under high-Pi conditions.

\subsection{Gene interactions}

The acid phosphatase gene family is a complex system that includes structural genes encoding different acid phosphatase and various regulatory factors. The interactions between phosphate transporters, acid phosphatase, and several important regulatory factors that modulate acid phosphatase transcription are described as follows.

Pho84 encodes a high-affinity phosphate transporter, located on the cytoplasmic membrane, which is used to transport the extracellular free inorganic phosphate particles that hydrolyzed by acid phosphatase to the body. Pho5 encodes a repressing acid phosphatase that releases phosphate from extracellular phosphate compounds and makes phosphate more easily be taken up by yeast [18]; Pho4 encodes a positive transcription factor that binds to regulatory sequences located on the upstream of the structural gene and then activates structural gene transcription, as shown in Figure 1; Pho85 has been shown to be a protein kinase, forms the Pho80-Pho85 cyclin-dependent kinase (CDK) complex by binding to Pho80 which has no kinase activity, then phosphorylates and inactivates Pho4, thereby blocks the expression of pho5 [19, 20 ]; Ph81 encodes a cyclin-dependent kinase inhibitor that can accept information from the external environment and act on Pho85-Pho80 under low phosphate conditions, leading Pho4 dephosphorylation [21].

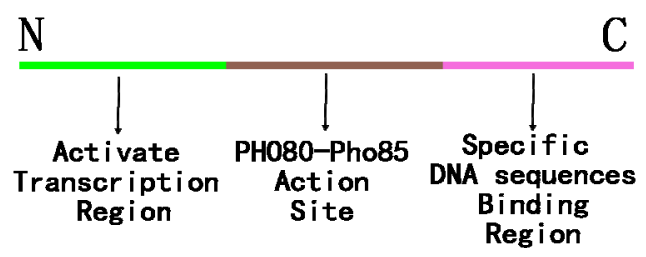

Figure 1. The structure of $\mathrm{PHO} 5$

\subsection{Protein regulation}

Under different external phosphorus environment, the expression of PHO pathway is different. When under low-phosphorus conditions, the PHO regulatory pathway is down-regulated. Pho81 keeps its original conformation and inactivates CDK complex Pho80Pho85 by binding it. Pho4 acts on the structural gene upstream activation sequence for Pho5 transcription and expression. And Pho84 is released at the same time. So, at this time, it is in a period of strong phosphorus absorption. When under high-phosphorus conditions, PHO regulatory pathway is up-regulated. Pho81 changes its original conformation so that cannot combine with CDK complex Pho80-Pho85. Pho4 is phosphorylated by
Pho80-Pho85, and export from the nucleus to the cytoplasm, thereby closing the expression of Pho5 and Pho84 genes. So, yeast cells cannot absorb phosphorus from the outside world at this time. Through this interaction between proteins, the dynamic balance of inorganic phosphorus in yeast cells is maintained [22]. The regulation process is shown in Figure 2.

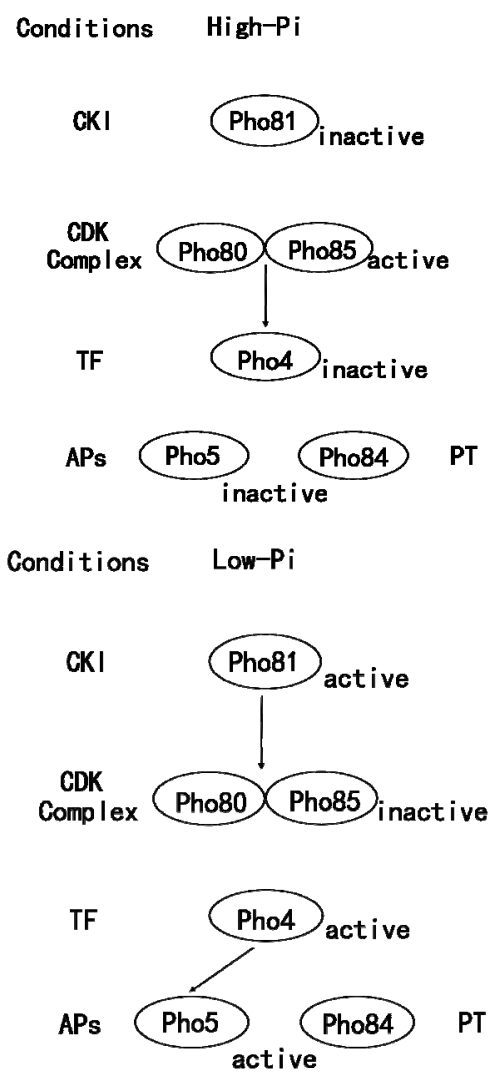

Figure 2. The regulation process under different conditions

\section{Mutants}

\subsection{The PHO mutants}

Hypothetically, if some phosphorus-respond gene in the PHO system are mutated, the yeast can release large amounts of acid phosphatase and phosphorus transporter even under high-phosphorus conditions, so that the phosphorus-removing properties of the yeast are not influenced by the external environmental concentration of phosphorus, which can great improve the phosphorus removal ability. This strains which have higher ability to remove phosphorus are called yeast mutant strains by changing its own phosphorus removal characteristics through EMS mutagens, site-directed gene mutations and other technical means.

Takashi Watanabe [23] obtained two yeast strains with excess phosphorus uptake capacity from brewery wastewater by gene mutation methods. Their phosphorus uptake capacity was about 2.2 to 3.5 times than ordinary yeasts, and they can remove $95 \%$ of soluble total phosphorus in sake waste water. The in-depth study found that the reason why these two strains can absorb 
phosphorus excessively is that the PHO pathway stress mode in cells which impacted by external phosphorus concentration has changed.

Takashi Watanabe [18] used mutagenic agents to mutagenize cell mutations to isolate mutants that can also secret large amounts of acid phosphatase in highphosphorus medium among laboratory-grown Saccharomyces cerevisiae, and the total soluble phosphorus removal rate was reached at $95 \%$, the polyphosphate released from the mutant strain was 3 to 5 times that of the raw yeast.

It can be demonstrated that the idea of changing the phosphorus-response gene sequence by certain technical means to improve the phosphorus removal characteristics of yeast cells is feasible. Because the cells unable to make an original reaction to the external environment, so that in a period of strong phosphorus absorption all the time.

\subsection{Polyphosphate}

Polyphosphate is a linear compound of phosphate and is the Pi reservoir for most organisms [24]. According to studies, about $40 \%$ of the phosphate in yeast accumulates in the form of polyphosphate, and $90 \%$ is located in the vacuole [25, 26]. Takashi Watanabe [18] treated yeast cells with a certain concentration of DAPI solution. After observation by fluorescence microscopy, it was found that yellow fluorescent polyphosphate particles were present in the vacuole. Elena V. Sambuk [16] analyzed gene expression levels in yeast cells on high and low Pi concentrations and found that the genes encoding vacuolar membrane integrin have similar expression levels to many genes in the PHO system and also are adjusted by $\mathrm{Pi}$ concentration. Through the determination of phosphate concentration in yeast, it was found that different PHO gene mutant strains have different polyphosphate concentration storage, demonstrating that the concentration of polyphosphate in yeast is regulated by the PHO system.

\section{Concluding remarks}

This is an exciting time in the phosphate removal field. Yeast occupies unique advantages in the removal of phosphorus from wastewater. However, due to the small proportion of yeast in activated sludge, few people currently use yeast for engineering practice. At the same time, studies have shown that the yeast excess sludge after removal of phosphorus is particularly easy to handle. By heating the sludge up to $70{ }^{\circ} \mathrm{C}$, the phosphorus in the cell can be eluted into the liquid phase and by adding $\mathrm{CaCl}_{2}$ to liquid phase, the phosphate and polyphosphate can be precipitated, which make phosphorus recovery become a particularly simple matter. Therefore, phosphorus removal from yeast wastewater can be greatly expected.

\section{Acknowledgement}

This work was financially supported by the National Science Foundation of China (51678276), the key research and development program of Shandong Province (2016CYJS07A03-3, 2016GSF117012, 2018GSF117026) and the Shandong Provincial Natural Science Foundation (ZR2018BEE040).

\section{References}

1. C. Niewersch, A.L.B. Bloch, S. Yüce, T. Melin, M. Wessling, Nanofiltration for the recovery of phosphorus-Development of a mass transport model , Desalination 346, 70-78(2014)

2. Z. Ying, N. Yuikawa, H. Nakatsuka, H. Maekawa, S. Harashima, Y. Nakanishi, Y. Kaneko, Core regulatory components of the PHO pathway are conserved in the methylotrophic yeast Hansenula polymorpha, Curr. Genet. 62, 595-605(2016)

3. M. Ikeh, Y. Ahmed, J. Quinn, Phosphate Acquisition and Virulence in Human Fungal Pathogens, Microorganisms 5, 48(2017)

4. K.K. Yadav, N. Singh, R. Rajasekharan, Responses to phosphate deprivation in yeast cells, Curr. Genet. 62, 301-307(2016)

5. E.M. Smith, Y.T. Prairie, Bacterial Metabolism and Growth Efficiency in Lakes: The Importance of Phosphorus Availability, Limnol. Oceanogr. 49, 137-147(2004)

6. M.A. Lachance, C.P. Kurtzman, J.W. Fell, V. Robert, J. Stalpers, T. Boekhout, M. BolotinFukuhara, K. Boundy-Mills, F. Rodrigues, P. Ludovico, Biodiversity and Ecophysiology of Yeasts, (Springer Berlin Heidelberg, 2006)

7. M. Yang, S. Zheng, Pollutant removal-oriented yeast biomass production from high-organicstrength industrial wastewater: A review, Biomass. Bioenerg. 64, 356-362(2014)

8. I. Borodina, J. Nielsen, Advances in metabolic engineering of yeast Saccharomyces cerevisiae for production of chemicals, Biotech. J. 9, 609620(2014)

9. N. Jin, Y. Jin, L.S. Weisman, Early protection to stress mediated by CDK-dependent PI3,5P2 signaling from the vacuole/lysosome, J. Cell. Biol. 216, 2075(2017)

10. C. Auesukaree, T. Homma, H. Tochio, M. Shirakawa, Y. Kaneko, S. Harashima, Intracellular phosphate serves as a signal for the regulation of the PHO pathway in Saccharomyces cerevisiae, J. Biol. Chem. 279, 17289-17294(2004)

11. B.R. Watts, S. Wittmann, M. Wery, C. Gautier, K. Kus, A. Birot, D.H. Heo, C. Kilchert, A. Morillon, L. Vasiljeva, Histone deacetylation promotes transcriptional silencing at facultative heterochromatin, Nucleic Acids Res. 232 (2018)

12. A. Toh-E, M. Ohkusu, H.M. Li, K. Shimizu, A. Takahashi-Nakaguchi, T. Gonoi, S. Kawamoto, Y. Kanesaki, H. Yoshikawa, M. Nishizawa, 
Identification of genes involved in the phosphate metabolism in Cryptococcus neoformans, Fungal Genet. Biol. 80, 19-30(2015)

13. M. Kretschmer, E. Reiner, G. Hu, N. Tam, D.L. Oliveira, M. Caza, H.Y. Ju, J. Kim, C.J. Kastrup, W.H. Jung, Defects in Phosphate Acquisition and Storage Influence Virulence of Cryptococcus neoformans, Infect. Immun. 82, 2697-2712(2014)

14. B.Z. He, X. Zhou, E.K. O'Shea, Evolution of reduced co-activator dependence led to target expansion of a starvation response pathway, Elife $\mathbf{6}$, (2017)

15. K. Romanowski, A. Zaborin, V. Valuckaite, R.J. Rolfes, T. Babrowski, C. Bethel, A. Olivas, O. Zaborina, J.C. Alverdy, Candida albicans isolates from the gut of critically Ill patients respond to phosphate limitation by expressing filaments and a lethal phenotype, Plos One 7, e30119(2012)

16. E.V. Sambuk, A.Y. Fizikova, V.A. Savinov, M.V. Padkina, Acid phosphatases of budding yeast as a model of choice for transcription regulation research, Enzyme Res. 2011, 356093(2011)

17. J.Y. Youn, H. Friesen, A.N. Nguyen Ba, W. Liang, V. Messier, M.J. Cox, A.M. Moses, B. Andrews, Functional Analysis of Kinases and Transcription Factors in Saccharomyces cerevisiae Using an Integrated Overexpression Library, G3 Genes. 7, 911-921(2017)

18. T. Watanabe, N. Ozaki, K. Iwashita, T. Fujii, H. Iefuji, Breeding of wastewater treatment yeasts that accumulate high concentrations of phosphorus, Appl. Microbiol. Biot. 80, 331-338(2008)

19. A. Kaffman, I. Herskowitz, R. Tjian, E.K. O'Shea, Phosphorylation of the transcription factor $\mathrm{PHO} 4$ by a cyclin-CDK complex, PHO80-PHO85, Science 263, 1153-1156(1994)

20. A. Tohe, K. Tanaka, Y. Uesono, R.B. Wickner, $\mathrm{PHO} 85$, a negative regulator of the $\mathrm{PHO}$ system, is a homolog of the protein kinase gene, CDC28, of Saccharomyces cerevisiae, Mol. Gen. Genet. 214, 162-164(1988)

21. K.R. Schneider, R.L. Smith, E.K. O'Shea, Phosphate-regulated inactivation of the kinase PHO80-PHO85 by the CDK inhibitor PHO81, Science 266, 122-126(1994)

22. Y. Oshima, The phosphate system in Saccharomyces cerevisiae, Genes Genet. Syst. 72, 323-334(1998)

23. T. Watanabe, K. Masaki, K. Iwashita, T. Fujii, H. Iefuji, Treatment and phosphorus removal from high-concentration organic wastewater by the yeast Hansenula anomala J224 PAWA, Bioresour. Technol. 99, 1781-1785(2009)

24. G.J.J. Kortstee, M.C.M.V. Loosdrecht, Inorganic Polyphosphates (Springer Berlin Heidelberg, 2005)

25. I.S. Kulaev, V.M. Vagabov, T.V. Kulakovskaya, The biochemistry of inorganic polyphosphates, Ergebn. Physiol. 73, 131-158(2005)
26. L. Lichko, T. Kulakovskaya, N. Pestov, I. Kulaev, Inorganic polyphosphates and exopolyphosphatases in cell compartments of the yeast Saccharomyces cerevisiae under inactivation of PPX1 and PPN1 genes, Biosci Rep. 26, 45-54(2006) 